\title{
New periodic solutions with a prescribed energy for a class of Hamiltonian systems
}

Fengying $\mathrm{Li}^{1}$, Ying $\mathrm{Lv}^{2^{*}}$ and Shiqing Zhang ${ }^{3}$

\section{"Correspondence:} ly0904@swu.edu.cn

${ }^{2}$ Department of Mathematics and

Statistics, Southwest University,

Chongqing, 400715, China

Full list of author information is

available at the end of the article

\begin{abstract}
We consider a class of second order Hamiltonian systems with a $C^{2}$ potential function. The existence of new periodic solutions with a prescribed energy is established by the use of constrained variational methods.

MSC: $34 \mathrm{C} 15 ; 34 \mathrm{C} 25 ; 58 \mathrm{~F}$

Keywords: second order Hamiltonian systems; $C^{2}$ periodic solutions; constrained variational minimizing methods
\end{abstract}

\section{Introduction}

In this paper, we examine the existence of periodic solutions for second order Hamiltonian systems

$$
\begin{aligned}
& \ddot{q}+V^{\prime}(q)=0, \\
& \frac{1}{2}|\dot{q}|^{2}+V(q)=h,
\end{aligned}
$$

with a fixed energy. The first major result in this direction we would like to highlight can be derived from the work of Benci [1], Gluck-Ziller [2], and Hayashi [3], which is based on the earlier work of Seifert [4] in 1948 and following the highly influential papers of Rabinowitz [5, 6] in 1978 and 1979. Utilizing the Jacobi metric and a very involved interplay between geodesic methods and algebraic topology, the following general theorem is established.

Theorem 1.1 Suppose $V \in C^{1}\left(\mathbb{R}^{n}, \mathbb{R}\right)$. If the potential well

$$
\left\{x \in \mathbb{R}^{n}: V(x) \leq h\right\}
$$

is bounded and non-empty, then the system (1.1)-(1.2) has a periodic solution with energy $h$. Furthermore, if

$$
V^{\prime}(x) \neq 0, \quad \forall x \in\left\{x \in \mathbb{R}^{n}: V(x)=h\right\},
$$

then the system (1.1)-(1.2) has a non-constant periodic solution with energy $h$.

(c) The Author(s) 2017. This article is distributed under the terms of the Creative Commons Attribution 4.0 International License (http://creativecommons.org/licenses/by/4.0/), which permits unrestricted use, distribution, and reproduction in any medium, provided you give appropriate credit to the original author(s) and the source, provide a link to the Creative Commons license, and indicate if changes were made. 
For the existence of multiple periodic solutions for (1.1)-(1.2) with compact energy surfaces, we can refer the reader to Groessen [7] and Long [8] and the references therein.

For the weakly attractive potential $V$ defined on an open subset $\Omega$ of $\mathbb{R}^{n}$, Ambrosetti and Coti Zelati [9] (Theorem 16.7) proved the following.

Theorem 1.2 Suppose $V \in C^{2}(\Omega, \mathbb{R})$ satisfies

$(V 10) 3\left\langle V^{\prime}(x), x\right\rangle+\left\langle V^{\prime \prime}(x) x, x\right\rangle \neq 0, \forall x \in \Omega$;

$(V 11)\left\langle V^{\prime}(x), x\right\rangle>0, \forall x \in \Omega$;

(V12) $\exists \alpha \in(0,2)$, such that $\left\langle V^{\prime}(x), x\right\rangle \geq-\alpha V(x), \forall x \in \Omega$;

(V13) $\exists \beta \in(0,2)$ and $r>0$ such that $\left\langle V^{\prime}(x), x\right\rangle \leq-\beta V(x), \forall 0<|x|<r$;

(V14) $G_{\infty} \geq 0$; where $G_{\infty}=\lim _{|x| \rightarrow \infty} \inf G(x), G(x)=\left[V(x)+\frac{1}{2}\left\langle V^{\prime}(x), x\right\rangle\right]$.

Then $\forall h<0$, the system (1.1)-(1.2) (referred to as $\left.\left(P_{h}\right)\right)$ has at least one non-constant weak periodic solution with the given energy $h$.

Using a simpler constrained variational minimizing method, we obtain the following result.

Theorem 1.3 Suppose $V \in C^{2}\left(\mathbb{R}^{n}, \mathbb{R}\right)$ and $h \in \mathbb{R}$ satisfy

$\left(V_{1}\right) \quad V(-q)=V(q)$

$\left(V_{2}\right)\left\langle V^{\prime}(q), q\right\rangle>0, \forall q \neq 0$;

$\left(V_{3}\right) 3\left\langle V^{\prime}(q), q\right\rangle+\left\langle V^{\prime \prime}(q) q, q\right\rangle>0, \forall q \neq 0$;

$\left(V_{4}\right) \exists \mu_{1}>0, \mu_{2} \geq 0$, such that $\left\langle V^{\prime}(q), q\right\rangle \geq \mu_{1} V(q)-\mu_{2}$;

$\left(V_{5}\right) \lim _{|q| \rightarrow \infty} \sup \left[V(q)+\frac{1}{2}\left\langle V^{\prime}(q), q\right\rangle\right] \leq A$;

$\left(V_{6}\right) \frac{\mu_{2}}{\mu_{1}}<h<A$.

Then the system (1.1)-(1.2) has at least one non-constant periodic solution with the given energy $h$.

Remark 1.4 Comparing Theorem 16.7 of Ambrosetti and Coti Zelati [9] with our Theorem 1.3, we notice that our condition $\left(V_{2}\right)$ corresponds to their $(V 11)$, our condition $\left(V_{3}\right)$ corresponds to their $(V 10)$, our condition $\left(V_{4}\right)$ corresponds to their $(V 12)$ and (V13), our conditions $\left(V_{5}\right)$ and $\left(V_{6}\right)$ correspond to their $(V 14)$. Since the potential in Theorem 16.7 of Ambrosetti and Coti Zelati has a singularity, but the potential in Theorem 1.3 has no singularity, the two theorems are essentially different.

Remark 1.5 Take for $V(x)$ the following $C^{\infty}$ function:

$$
\begin{aligned}
& V(x)=e^{\frac{-1}{|x|}}, \quad \forall x \neq 0 ; \\
& V(0)=0 .
\end{aligned}
$$

Then $V(x)$ satisfies $\left(V_{1}\right)-\left(V_{5}\right)$ in Theorem 1.3 if we take $\mu_{1}=\mu_{2}>0$ and $A=1$, but $\left(V_{6}\right)$ does not hold.

Proof of Theorem 1.3 We verify $\left(V_{1}\right)-\left(V_{5}\right)$ by calculation:

(1) It is obvious for $\left(V_{1}\right)$. 
(2) For $\left(V_{2}\right)$ and $\left(V_{3}\right)$, we notice that

$$
\begin{aligned}
& \left\langle V^{\prime}(x), x\right\rangle=\frac{1}{|x|} e^{\frac{-1}{|x|}}>0, \quad \forall x \neq 0, \\
& \left\langle V^{\prime \prime}(x) x, x\right\rangle=e^{\frac{-1}{|x|}}\left(\frac{-2}{|x|}+\frac{1}{|x|^{2}}\right), \\
& 3\left\langle V^{\prime}(x), x\right\rangle+\left\langle V^{\prime \prime}(x) x, x\right\rangle=e^{\frac{-1}{|x|}}\left(\frac{1}{|x|}+\frac{1}{|x|^{2}}\right)>0, \quad \forall x \neq 0 .
\end{aligned}
$$

(3) For $\left(V_{4}\right)$, we set

$$
w(x)=\left(\frac{1}{|x|}-\mu_{1}\right) e^{\frac{-1}{|x|}} ; \quad x \neq 0, w(0)=0 .
$$

We will prove $w(x)>-\mu_{1}$; in fact,

$$
w^{\prime}(x)=\left[\frac{1}{|x|}-\left(1+\mu_{1}\right)\right] \frac{x}{|x|^{3}} e^{\frac{-1}{|x|}} ; \quad x \neq 0, w^{\prime}(0)=0 .
$$

From $w^{\prime}(x)=0$, we have $x=-\frac{1}{1+\mu_{1}}$ or 0 or $\frac{1}{1+\mu_{1}}$.

It is easy to see that $w(x)$ is strictly increasing on $\left(-\infty,-\frac{1}{1+\mu_{1}}\right]$ and $\left[0, \frac{1}{1+\mu_{1}}\right]$, but strictly decreasing on $\left[\frac{-1}{1+\mu_{1}}, 0\right]$ and $\left[\frac{1}{1+\mu_{1}},+\infty\right)$. We notice that

$$
\lim _{|x| \rightarrow+\infty} w(x)=-\mu_{1}
$$

and

$$
w(0)=0 \text {. }
$$

So

$$
w(x)>-\mu_{1}
$$

When we take $\mu_{2}=\mu_{1}>0$, $\left(V_{4}\right)$ holds.

(4) For $\left(V_{5}\right)$, we have

$$
\begin{aligned}
& V(x)+\frac{1}{2}\left\langle V^{\prime}(x), x\right\rangle=e^{\frac{-1}{|x|}}\left(1+\frac{1}{2} \frac{1}{|x|}\right)<1, \quad \forall x \neq 0 ; \\
& V(0)+\frac{1}{2}\left\langle V^{\prime}(0), 0\right\rangle=0 .
\end{aligned}
$$

Corollary 1.6 Given $a>0, n \in \mathbb{N}$, define $V(x)=a|x|^{2 n}+e^{\frac{-1}{|x|}}, x \neq 0 ; V(0)=0$. Then, for $h>1$, the system (1.1)-(1.2) has at least one non-constant periodic solution with the given energy $h$.

Remark 1.7 The potential $V(x)=e^{\frac{-1}{|x|}}, \forall x \neq 0 ; V(0)=0$ in Remark 1.5 is noteworthy since the potential function is non-convex and bounded which satisfies neither of the conditions of Theorems 1.1, Offin's geometrical conditions [10], nor Berg-PasquottoVandervorst's complex topological assumptions [11]. For this potential, the potential well 
$\left\{x \in \mathbb{R}^{n}: V(x) \leq h\right\}$ is a bounded set if $h<1$, but for $h \geq 1$ it is $\mathbb{R}^{n}$ - an unbounded set. We also notice that the symmetrical condition on the potential simplified our Theorem 1.2 and its proof. It would be interesting to obtain non-constant periodic solutions when the symmetrical condition is deleted.

\section{A few lemmas}

Let

$$
H^{1}=W^{1,2}\left(\mathbb{R}_{\mathrm{per}}, \mathbb{R}^{n}\right)=\left\{u: \mathbb{R} \rightarrow \mathbb{R}^{n}, u(t+1)=u(t), u \in L^{2}[0,1], \dot{u} \in L^{2}[0,1]\right\}
$$

denotes the periodic functional space of period 1 . Then the standard $H^{1}$ norm is

$$
\|u\|=\|u\|_{H^{1}}=\left(\int_{0}^{1}|\dot{u}|^{2} d t\right)^{1 / 2}+\left(\int_{0}^{1}|u|^{2} d t\right)^{1 / 2} .
$$

Lemma 2.1 ([12]) For $u \in H^{1}$, define

$$
\begin{aligned}
& g(u)=\int_{0}^{1}\left[V(u)+\frac{1}{2}\left\langle V^{\prime}(u), u\right\rangle\right] d t, \\
& M=\left\{u \in H^{1}: g(u)=h\right\} .
\end{aligned}
$$

For $u, v \in H^{1}$ and $s \in \mathbb{R}$, let

$$
\phi(s)=g(u+s v) .
$$

Then

$$
\phi^{\prime}(0)=\left\langle g^{\prime}(u), v\right\rangle=\frac{1}{2} \int_{0}^{1}\left\{3\left\langle V^{\prime}(u), v\right\rangle+\left\langle V^{\prime \prime}(u) v, u\right\rangle\right\} d t
$$

and

$$
\left\langle g^{\prime}(u), u\right\rangle=\frac{1}{2} \int_{0}^{1}\left\{3\left\langle V^{\prime}(u), u\right\rangle+\left\langle V^{\prime \prime}(u) u, u\right\rangle\right\} d t
$$

therefore, if $\left(V_{3}\right)$ holds, then on $M, g^{\prime}(u) \neq 0$, which implies $M$ is a $C^{1}$ manifold with codimension 1 in $H^{1}$.

Let

$$
f(u)=\frac{1}{4} \int_{0}^{1}|\dot{u}|^{2} d t \int_{0}^{1}\left\langle V^{\prime}(u), u\right\rangle d t
$$

and $\tilde{u} \in M$ such that $f^{\prime}(\tilde{u})=0$ and $f(\widetilde{u})>0$. Set

$$
\frac{1}{T^{2}}=\frac{\int_{0}^{1}\left\langle V^{\prime}(\widetilde{u}), \tilde{u}\right\rangle d t}{\int_{0}^{1}|\dot{\vec{u}}|^{2} d t} .
$$

If $\left(V_{2}\right)$ holds, then $\widetilde{q}(t)=\widetilde{u}(t / T)$ is a non-constant $T$-periodic solution for (1.1)-(1.2). 
When the potential is even, then by Palais' symmetrical principle [13] and Lemma 2.1 we have the following.

Lemma $2.2([12])$ Let

$$
F=\left\{u \in M: u\left(t+\frac{1}{2}\right)=-u(t)\right\}
$$

and suppose $\left(V_{1}\right)-\left(V_{3}\right)$ hold. If $\widetilde{u} \in F$ is such that $f^{\prime}(\widetilde{u})=0$ and $f(\widetilde{u})>0$, then $\widetilde{q}(t)=\widetilde{u}\left(\frac{t}{T}\right)$ is a non-constant T-periodic solution for (1.1)-(1.2); in addition, we have

$$
\forall u \in F, \quad \int_{0}^{1} u(t) d t=0
$$

Wirtinger's inequality [14] implies

$$
\int_{0}^{1}|\dot{u}|^{2} d t \geq(2 \pi)^{2} \int_{0}^{1}|u|^{2}
$$

from which it follows that $\left(\int_{0}^{1}|\dot{u}|^{2} d t\right)^{1 / 2}$ is an equivalent norm for the space $H^{1}$.

Lemma 2.3 Let $X$ be a Banach space and $F \subset X$ a weakly closed subset. Suppose $\Phi$ defined on $F$ is Gateaux-differentiable, weakly lower semi-continuous and bounded from below on F. Suppose further that $\Phi$ satisfies the following (WPS) inf $\Phi, F_{\text {condition: }}$

- If $\left\{x_{n}\right\} \subset F$ such that $\Phi\left(x_{n}\right) \rightarrow c$ and $\left\|\Phi^{\prime}\left(x_{n}\right)\right\| \rightarrow 0$, then $\left\{x_{n}\right\}$ has a weakly convergent subsequence.

Then $\Phi$ attains its infimum on $F$.

Proof By Ekeland's variational principle $[15,16]$, we know that there is a sequence $\left\{x_{n}\right\} \subset F$ satisfying

$$
\Phi\left(x_{n}\right) \rightarrow \inf \Phi \text { and } \quad\left\|\Phi^{\prime}\left(x_{n}\right)\right\| \rightarrow 0
$$

Since $\Phi$ satisfies the (WPS) $)_{\text {inf } \Phi, F}$ condition, $\left\{x_{n}\right\}$ has a weakly convergent subsequence which as a weak limit $x$. Because $F \subset X$ is a weakly closed subset, we have $x \in F$. Finally, by the weakly lower semi-continuous assumption on $\Phi$, we conclude that $\Phi$ attains its infimum on $F$.

\section{The proof of Theorem 1.3}

We prove Theorem 1.3 by the following sequence of lemmas. In the following, $f$ and $F$ are defined as in (2.1) and (2.2), respectively.

Lemma 3.1 If $\left(V_{1}\right)-\left(V_{6}\right)$ hold, then, for any given $c>0, f$ satisfies the $(P S)_{c, F}$ condition; that is, if $\left\{u_{n}\right\} \subset F$ satisfies

$$
f\left(u_{n}\right) \rightarrow c>0 \text { and }\left.\quad f\right|_{F} ^{\prime}\left(u_{n}\right) \rightarrow 0
$$

then $\left\{u_{n}\right\}$ has a strongly convergent subsequence. 
Proof We first prove that under our assumptions the constrained set $F \neq \emptyset$. For any given $u \in H^{1}$ satisfying $u(t) \neq 0, \forall t \in[0,1]$ and for $a>0$, let

$$
g_{u}(a)=g(a u)=\int_{0}^{1}\left[V(a u)+\frac{1}{2}\left\langle V^{\prime}(a u), a u\right\rangle\right] d t .
$$

By the assumption $\left(V_{3}\right)$, we have

$$
\frac{d}{d a} g_{u}(a)>0
$$

and so $g_{u}$ is strictly increasing. Since $V \in C^{2}$, we know that, for any given $a>0$,

$$
\left[V(a u(t))+\frac{1}{2}\left\langle V^{\prime}(a u(t)), a u(t)\right\rangle\right]
$$

is uniformly continuous on $[0,1]$.

Hence by $\left(V_{5}\right)$, we have

$$
\lim _{a \rightarrow+\infty} g_{u}(a) \leq \int_{0}^{1} \lim _{a \rightarrow+\infty} \sup \left[V(a u)+\frac{1}{2}\left\langle V^{\prime}(a u), a u\right\rangle\right] d t \leq A .
$$

By $\left(V_{4}\right)$, we notice that

$$
g_{u}(0)=V(0) \leq \frac{\mu_{2}}{\mu_{1}} .
$$

Since $\frac{\mu_{2}}{\mu_{1}}<h<A$, we see that the equation $g_{u}(a)=h$ has a unique solution $a(u)$ with $a(u) u \in M$.

$$
\text { By } f\left(u_{n}\right) \rightarrow c \text {, we have }
$$

$$
\frac{1}{4} \int_{0}^{1}\left|\dot{u}_{n}(t)\right|^{2} d t \cdot \int_{0}^{1}\left\langle V^{\prime}\left(u_{n}\right), u_{n}\right\rangle d t \rightarrow c,
$$

and by $\left(V_{4}\right)$ we see that

$$
h=\int_{0}^{1}\left[V\left(u_{n}\right)+\frac{1}{2}\left\langle V^{\prime}\left(u_{n}\right), u_{n}\right\rangle\right] d t \leq\left(\frac{1}{\mu_{1}}+\frac{1}{2}\right) \int_{0}^{1}\left\langle V^{\prime}\left(u_{n}\right), u_{n}\right\rangle d t+\frac{\mu_{2}}{\mu_{1}} .
$$

By (3.6) and (3.7), we have

$$
\int_{0}^{1}\left\langle V^{\prime}\left(u_{n}\right), u_{n}\right\rangle d t \geq \frac{h-\frac{\mu_{2}}{\mu_{1}}}{\frac{1}{2}+\frac{1}{\mu_{1}}} .
$$

Condition $\left(V_{6}\right)$ provides $h>\frac{\mu_{2}}{\mu_{1}}$. Then (3.6) and (3.8) imply $\int_{0}^{1}\left|\dot{u}_{n}(t)\right|^{2} d t$ is bounded and $\left\|u_{n}\right\|=\left\|\dot{u}_{n}\right\|_{L^{2}}$ is bounded.

We know that $H^{1}$ is a reflexive Banach space, so $\left\{u_{n}\right\}$ has a weakly convergent subsequence; furthermore, by the embedding theorem the weakly convergent subsequence also uniformly converges to some $u \in H^{1}$. The standard argument can show that $\left\{u_{n}\right\}$ has a subsequence which converges under the $H^{1}$ norm. We omit the details of this standard demonstration. 
Lemma 3.2 $f(u)$ is weakly lower semi-continuous on $F$.

Proof For any $u_{n} \subset F$ with $u_{n} \rightarrow u$, by Sobolev's embedding theorem we have the uniform convergence

$$
\left|u_{n}(t)-u(t)\right|_{\infty} \rightarrow 0
$$

Since $V \in C^{1}\left(\mathbb{R}^{n}, \mathbb{R}\right)$, we have

$$
\left|V\left(u_{n}(t)\right)-V(u(t))\right|_{\infty} \rightarrow 0 .
$$

By the weakly lower semi-continuity of the norm, we see that

$$
\liminf \left[\int_{0}^{1}\left|\dot{u}_{n}\right|^{2} d t\right]^{\frac{1}{2}} \geq\left(\int_{0}^{1}|\dot{u}|^{2} d t\right)^{\frac{1}{2}}
$$

and so

$$
\liminf \left(\int_{0}^{1}\left|\dot{u}_{n}\right|^{2} d t\right) \geq \int_{0}^{1}|\dot{u}|^{2} d t .
$$

Then

$$
\begin{aligned}
\liminf f\left(u_{n}\right) & =\liminf \frac{1}{4} \int_{0}^{1}\left|\dot{u}_{n}\right|^{2} d t \int_{0}^{1}\left\langle V^{\prime}\left(u_{n}\right), u_{n}\right\rangle d t \\
& \geq \frac{1}{4} \int_{0}^{1}|\dot{u}|^{2} d t \int_{0}^{1}\left\langle V^{\prime}(u), u\right\rangle d t=f(u) .
\end{aligned}
$$

Lemma 3.3 $F$ is a weakly closed subset in $H^{1}$.

Proof This follows easily from Sobolev's embedding theorem and $V \in C^{1}\left(\mathbb{R}^{n}, \mathbb{R}\right)$.

Lemma 3.4 The functional $f(u)$ has a positive lower bound on $F$.

Proof By the definitions of $f(u), F$, and the assumption $\left(V_{2}\right)$, we have

$$
f(u)=\frac{1}{4} \int_{0}^{1}|\dot{u}|^{2} d t \int_{0}^{1}\left\langle V^{\prime}(u), u\right\rangle d t \geq 0, \quad \forall u \in F .
$$

We claim further that

$$
\inf f(u)>0
$$

otherwise, $\left(V_{2}\right)$ implies $u(t)=$ const, and by the symmetrical property $u(t+1 / 2)=-u(t)$ we have $u(t)=0, \forall t \in \mathbb{R}$. But assumptions $\left(V_{4}\right)$ and $\left(V_{6}\right)$ imply

$$
V(0) \leq \frac{\mu_{2}}{\mu_{1}}<h,
$$

which contradicts the definition of $F$ since $V(0)=h$ if we have $0 \in F$. Now by Lemmas 3.13.4 and Lemma 2.3, we see that $f(u)$ attains the infimum on $F$ and we know that the minimizer is non-constant. 
Competing interests

The authors declare that no competing interests exist.

\section{Authors' contributions}

The authors contributed equally to this paper. All authors read and approved the final manuscript.

\section{Author details}

${ }^{1}$ School of Economic Mathematics, Southwestern University of Finance and Economics, Chengdu, 61130, China.

${ }^{2}$ Department of Mathematics and Statistics, Southwest University, Chongqing, 400715, China. ${ }^{3}$ Department of Mathematics, Sichuan University, Chengdu, 610064, China.

\section{Acknowledgements}

The authors sincerely thank the editor and the referees for their many valuable comments and suggestions. Shiqing Zhang and Fengying Li were partially supported by NSFC (11671278). Ying Lv was partially supported by NSFC

(11601438).

Received: 30 November 2016 Accepted: 22 February 2017 Published online: 09 March 2017

\section{References}

1. Benci, V: Closed geodesics for the Jacobi metric and periodic solutions of prescribed energy of natural Hamiltonian systems. Ann. Inst. Henri Poincaré, Anal. Non Linéaire 1, 401-412 (1984)

2. Gluck, H, Ziller, W: Existence of periodic motions of conservative systems. In: Bombieri, E (ed.) Seminar on Minimal Submanifolds. Princeton University Press, Princeton (1983)

3. Hayashi, K. Periodic solutions of classical Hamiltonian systems. Tokyo J. Math 6, 473-486 (1983)

4. Seifert, H: Periodische Bewegungen mechanischer Systeme. Math. Z. 51, 197-216 (1948)

5. Rabinowitz, PH: Periodic solutions of Hamiltonian systems. Commun. Pure Appl. Math. 31, 157-184 (1978)

6. Rabinowitz, PH: Periodic solutions of a Hamiltonian systems on a prescribed energy surface. J. Differ. Equ. 33, 336-352 (1979)

7. Van Groesen, EWC: Analytical mini-max methods for Hamiltonian break orbits with a prescribed energy. J. Math. Anal. Appl. 132, 1-12 (1988)

8. Long, Y: Index Theory for Symplectic Paths with Applications. Birkhäuser, Basel (2002)

9. Ambrosetti, A, Coti Zelati, V: Periodic Solutions of Singular Lagrangian Systems. Birkhäuser, Basel (1993)

10. Offin, D: A class of periodic orbits in classical mechanics. J. Differ. Equ. 66, 90-117 (1987)

11. Berg, J, Pasquotto, F, Vandervorst, R: Closed characteristics on non-compact hypersurfaces in $\mathbb{R}^{2 n}$. Math. Ann. 343, 247-284 (2009)

12. Ambrosetti, A, Coti Zelati, V: Closed orbits of fixed energy for singular Hamiltonian systems. Arch. Ration. Mech. Anal. $112,339-362(1990)$

13. Palais, R: The principle of symmetric criticality. Commun. Math. Phys. 69, 19-30 (1979)

14. Mawhin, J, Willem, M: Critical Point Theory and Applications. Springer, Berlin (1989)

15. Ekeland, I: On the variational principle. J. Math. Anal. Appl. 47, 324-353 (1974)

16. Ekeland, I: Nonconvex minimization problems. Bull. Am. Math. Soc. (N.S.) 1(3), 443-474 (1979)

\section{Submit your manuscript to a SpringerOpen ${ }^{\circ}$ journal and benefit from:}

- Convenient online submission

Rigorous peer review

- Immediate publication on acceptance

- Open access: articles freely available online

- High visibility within the field

- Retaining the copyright to your article 